\title{
Monte Carlo Ray Tracing Based Sensitivity Analysis of the Atmospheric and the Ocean Parameters on Top of the Atmosphere Radiance
}

\author{
Kohei Arai ${ }^{1}$ \\ Graduate School of Science and Engineering \\ Saga University \\ Saga City, Japan
}

\begin{abstract}
Monte Carlo Ray Tracing: MCRT based sensitivity analysis of the geophysical parameters (the atmosphere and the ocean) on Top of the Atmosphere: TOA radiance in visible to near infrared wavelength regions is conducted. As the results, it is confirmed that the influence due to the atmosphere is greater than that of the ocean. Scattering and absorption due to aerosol particles and molecules in the atmosphere is major contribution followed by water vapor and ozone while scattering due to suspended solid is dominant contribution for the ocean parameters.
\end{abstract}

Keywords-Monte Carlo Ray Tracing; radiative transfer; scattering and absorption; geophysical parameters (the atmosphere and the ocean).

\section{INTRODUCTION}

It is not easy to solve Radiative Transfer Equation: RTE when the RTE includes radiative transfer in the ocean. There are some widely used RTE software codes, 6S, MODTRAN, and the others. These RTE models do not take into account the radiative transfer in the ocean; there are some RTE models for the RTE models for the ocean, though. RTE model proposed here is based on Monte Carlo Ray Tracing: MCRT model [1]. Therefore, it is assumed any materials, particles, molecules, and the others in the ocean.

Considerable geophysical parameters of the atmosphere and the ocean are assumed for investigation of sensitivity of the geophysical parameters on the Top of the Atmosphere: TOA radiance. The following section describes the proposed method for sensitivity analysis including MCRT simulation model. Preliminary simulation results are described together with the simulation results for sensitivity analysis followed by conclusion with some discussions.

\section{PROpOSED METHOD}

\section{A. Monte Carlo Ray Tracing Simulation Model}

Illustrative view of the proposed MCRT simulation model is shown in Figure 1. Photon from the sun is input from the top of the atmosphere (the top of the simulation cell). Travel length of the photon is calculated with optical depth of the atmospheric molecule and that of aerosol.

There are two components in the atmosphere; molecule and aerosol particles while three are also two components, water and particles; suspended solid and phytoplankton in the ocean. When the photon meets molecule or aerosol (the meeting probability with molecule and aerosol depends on their optical depth), then the photon scattered in accordance with scattering properties of molecule and aerosol [2].

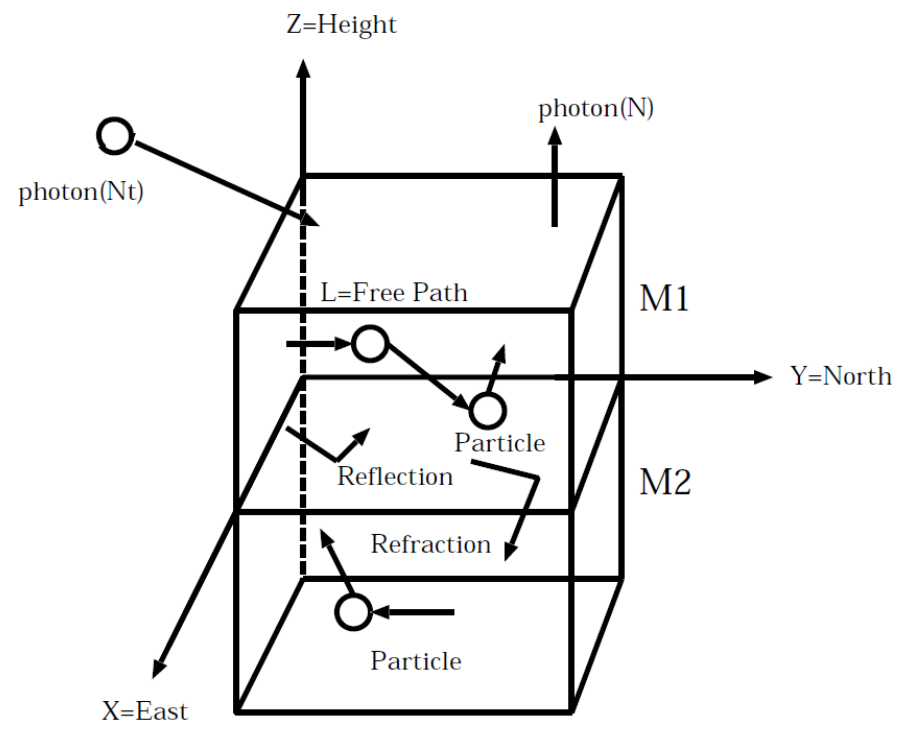

Figure 1. Proposed MCRT simulation model

For simplifying the calculations of the atmospheric influences, it is assumed that the atmosphere containing only molecules and aerosols. Thus the travel length of the photon at once, $L$ is expressed with equation (1).

$L=L_{0} \mathrm{RND}(i)$

$L_{0}=Z_{\max } / \tau$

where $Z_{\text {max }} \tau, \operatorname{RND}(i)$ are maximum length, altitude of the atmosphere, optical depth, and $i$-th random number, respectively. In this equation, $\tau$ is optical depth of molecule or aerosol. The photon meets molecule when the random number is greater than $\tau$.

Meanwhile, if the random number is less than $\tau$, then the photon meats aerosol. The photon is scattered at the molecule or aerosol to the direction which is determined with the phase function and with the rest of the travel length of the photon. 
Reflection, transpiration, and refraction of the photon at the sea surface are followed by Fresnell law [3] and Snell law [3] as shown in Figure 2.

In three dimensional simulation cells, photon direction has to be changed when the photon meets aerosol particle, molecule, ocean surface, suspended solid, phytoplankton, and so on in accordance with the rotation matrix shown in equation (3) and in Figure 3. In accordance with the number which put at the top left corner, the direction is changed.

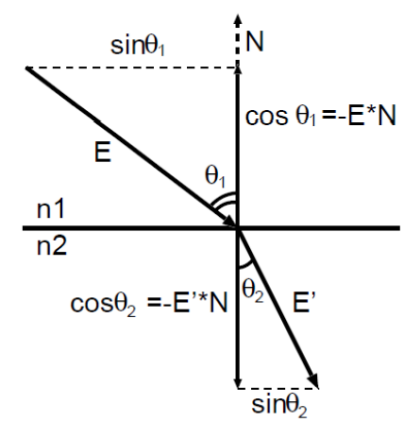

Figure 2. Two dimensional expression of reflection, transpiration, and refraction of the photon at the sea surface

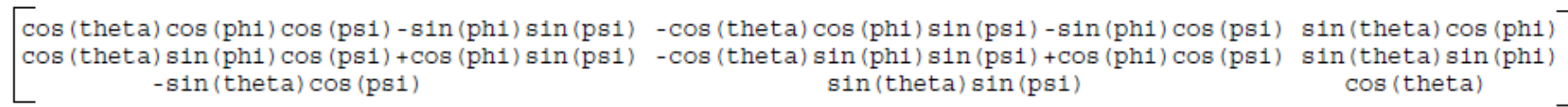
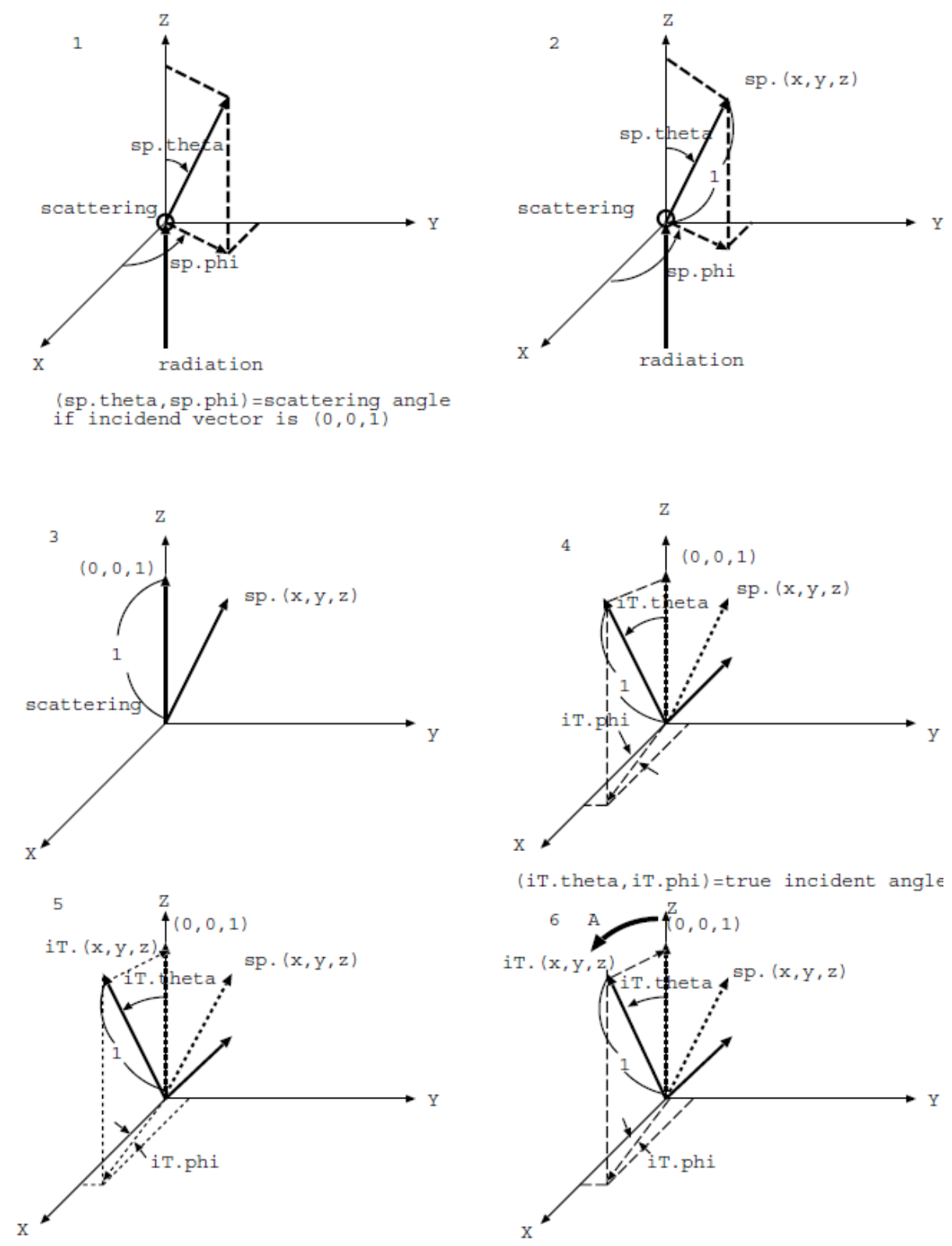

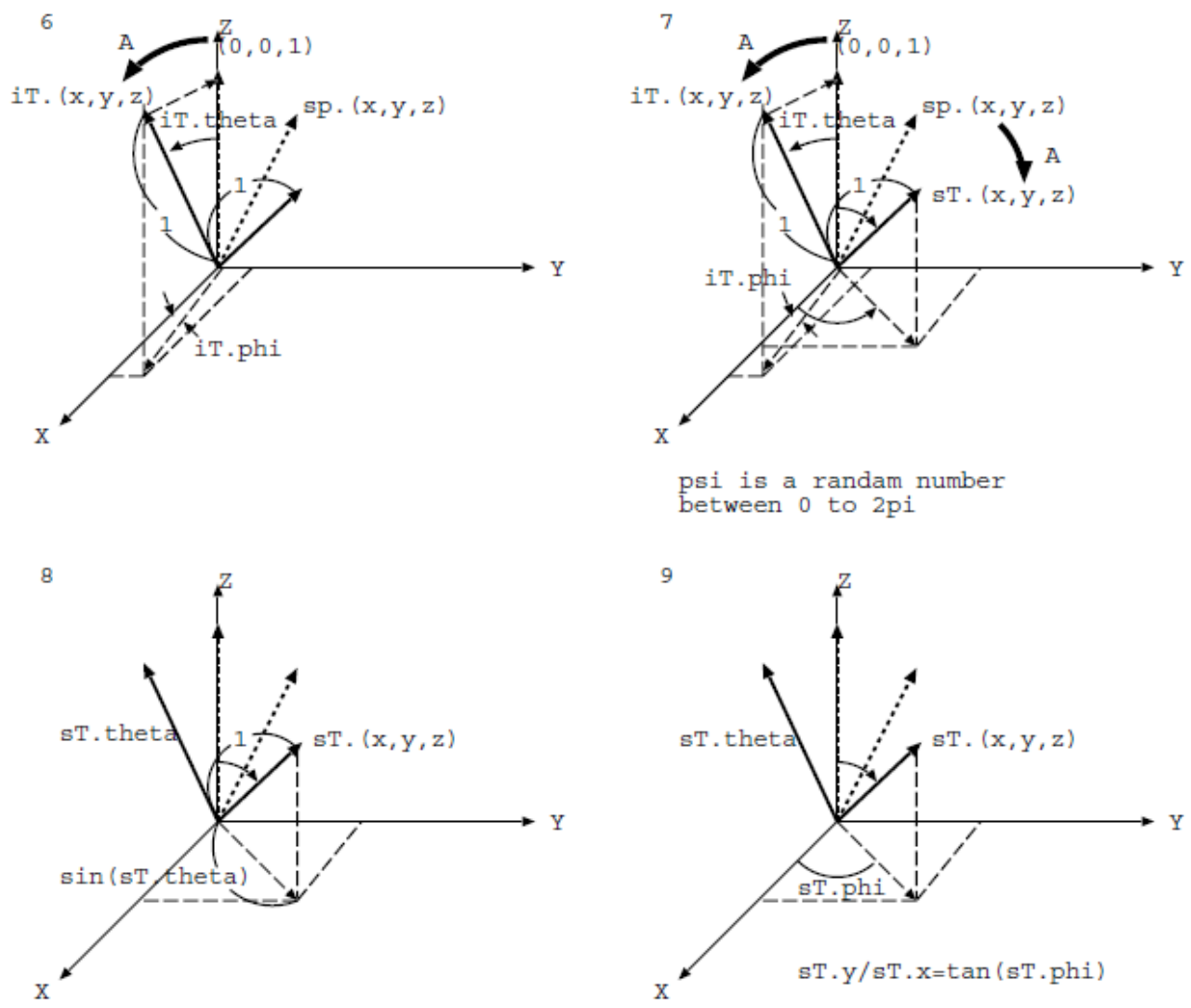

Figure 3. Process flow for photon direction changes when the photon in concern meets aerosol particle, molecule, ocean surface, suspended solid, phytoplankton, and so on

Where phi denote azimuth and psi denote elevation angles. In Figure 3, particle is situated in the center (origin of the three dimensional coordinate system). The phone comes from the bottom and meets with the particle in concern. The scattering angle is expressed as a function of sp.theta and sp.phi is the incident vector is $(0,0,1)$ while the true incident angle is a function of iT.theta and iT.phi in this figure. Then rotation matrix is reduced as shown in equation (3). This process flow is same for reflection, refraction, and scattering.

The scattering property is called as phase function. In the visible to near infrared wavelength region, the scattering by molecule is followed by Rayleigh scattering law [3] while that by aerosol is followed by Mie scattering law [3]. Example of phase function of Mie scattering is shown in Figure 4 (a) while that of Rayleigh scattering is shown in Figure 4 (b). In the figure, scattering angle is defined as the angle between incidence and reflected angle from the particle. These phase functions can be calculated with Mie Code in the MODerate resolution atmospheric TRANsmission; MODTRAN ${ }^{1}$.

\section{B. Top of the Atmosphere: TOA Radiance Calculation}

If the photon reaches on the wall of the simulation cell, the photon disappears at the wall and it appears from the corresponding position on the opposite side wall. Then it travels with the rest of travel length. Eventually, the photons which are reached at the top of the atmosphere are gathered with the Instantaneous Field of View: IFOV of the Visible to Near Infrared Radiometer: VNIR onboard satellite. At sensor radiance, $\mathrm{I}^{+}$with direction and IFOV of $\mu, \mu_{0}$ can be calculated with equation (4)

$$
I^{+}\left(\mu, \mu_{0}\right)=I N^{+}\left(\mu, \mu_{0}\right) / N_{\text {total }}
$$

where $N^{+}$is the number of photons which are gathered by VNIR, $N_{\text {total }}$ denotes the number of photons input to the simulation cell. Also I denotes extraterrestrial irradiance at the top of the atmosphere.

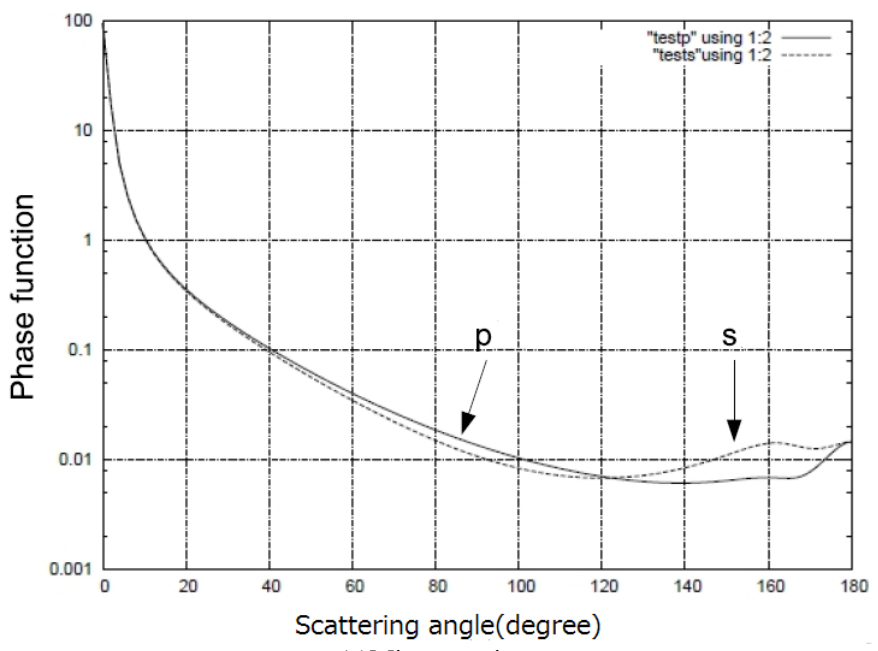

(a)Mie scattering

\footnotetext{
${ }^{1}$ http://modtran.org/
} 


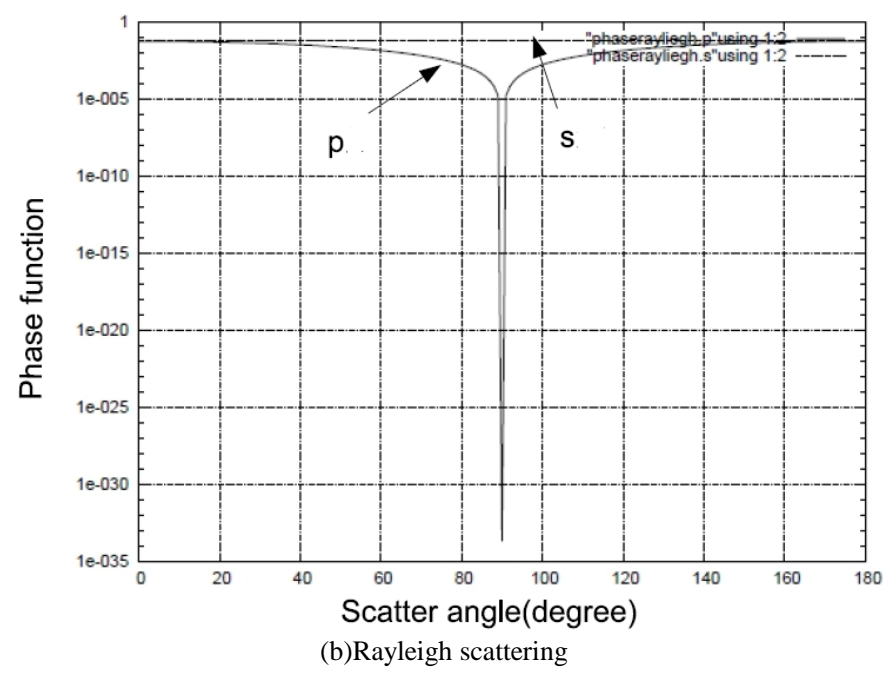

Figure 4. Phase functions for Mie and Rayleigh scattering

III. EXPERIEMNTS

\section{A. Preliminary Simulations}

Preliminary simulation results are shown in Figure 5.

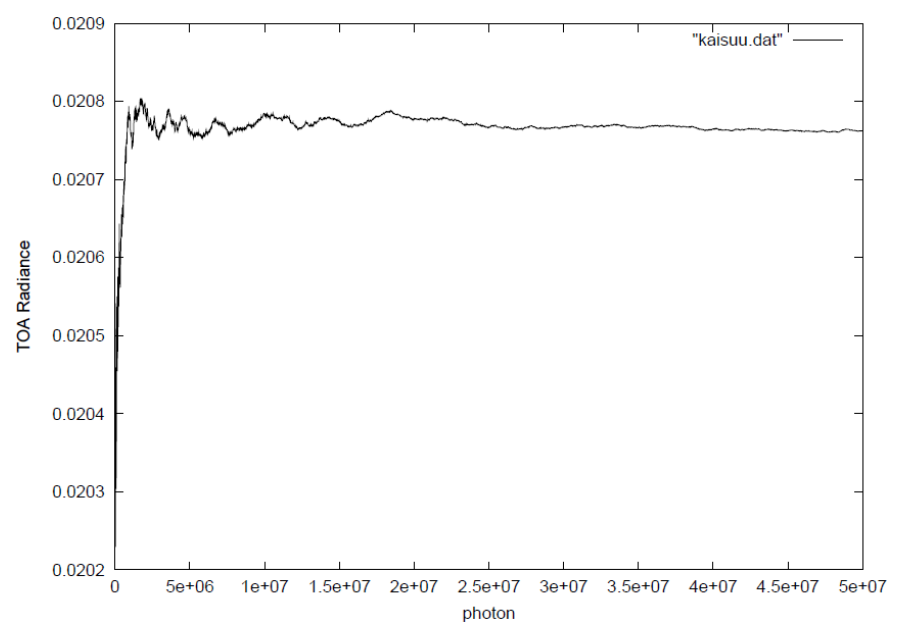

(a)TOA radiance

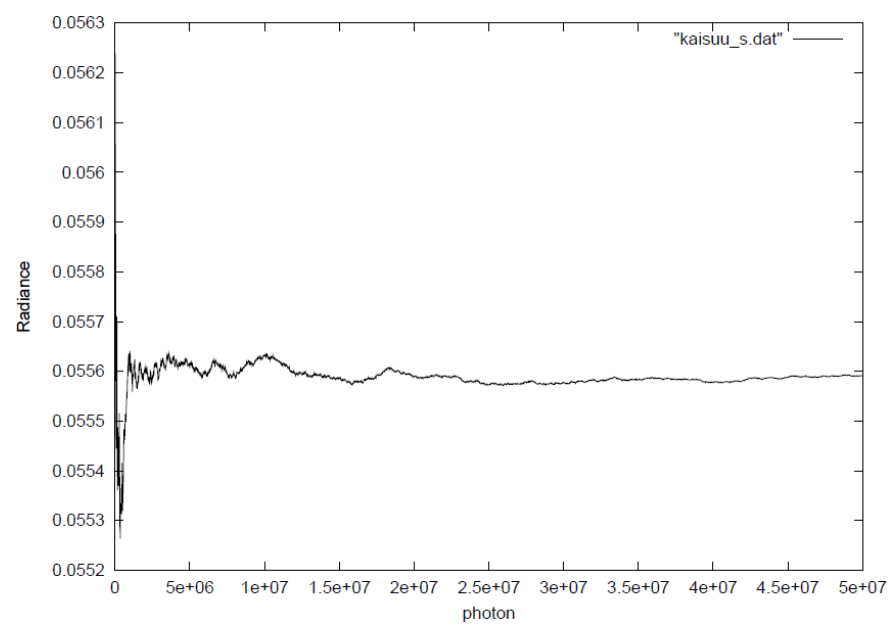

(b)Water leaving radiance

Figure 5. Examples of the preliminary simulation
In the preliminary simulation study, TOA radiance and water leaving radiance is calculated when the number of photons is changed from 1 to 50 million. The size of simulation cell is defined as $50 \mathrm{~km}$ by $50 \mathrm{~km}$ by $50 \mathrm{~km}$ for the atmosphere while that is set as $50 \mathrm{~km}$ by $50 \mathrm{~km}$ by $500 \mathrm{~m}$ (in depth) for the ocean. Wavelength is set at $500 \mathrm{~nm}$ while the solar azimuth and zenith angles are set at 90 and 30 degrees. All the parameters required for the simulation are set as follows,

Optical depth of suspended solid: 0.03

Molecule optical depth: 0.25

Aerosol optical depth: 0.3

Single scattering albedo of suspended solid: 0.3

Single scattering albedo of molecule in the ocean: 0.15

As the results, it is found that water leaving radiance is almost twice much greater than that of TOA radiance. Also TOA radiance includes pass-radiance (the photons are scattered in the atmosphere and then come out from the top of the atmosphere without reaching the ocean surface). Therefore, less than $1 / 10$ of small number of photons are come out from the top of the atmosphere from the ocean surface in comparison to the photons which are come out from the atmosphere from the atmosphere. Both TOA and water leaving radiance are saturated at the number of photons is around 30 million. Therefore, the number of photons is set at 30 million for the detailed simulation study on sensitivity analysis.

\section{B. Sensitivity Analysis}

Radiance_S and Radiance_T denote water leaving radiance and TOA radiance excluding the contribution due to scattering component in the atmosphere. Meanwhile TOA radiance includes all the contributions from the atmosphere and the ocean. Figure 6 (a) shows these radiances as a function of solar zenith angle. Default parameters for this simulation is as follows,

Optical depth of suspended solid: 0.015

Molecule optical depth: 0.15

Aerosol optical depth: 0.2

Single scattering albedo of suspended solid: 0.6

Single scattering albedo of molecule in the ocean: 0.3

All the parameters are set as default except optical depth of suspended solid. Then the simulation result of TOA radiance, water leaving radiance and TOA radiance excluding scattering component in the atmosphere as a function of optical depth of suspended solid is obtained and is shown in Figure 6 (b). Meanwhile, those radiances as functions of oceanic molecule optical depth, atmospheric molecule optical depth, aerosol optical depth, single scattering albedo of suspended solid, and single scattering albedo of molecule in the ocean are shown in Figure 6 (c), (d), (e), (f), and (g), respectively. From these figures, it is found that TOA radiance is not so sensitive to the suspended solid optical depth, oceanic molecule optical depth, atmospheric molecule optical depth, aerosol optical depth, single scattering albedo of suspended solid and is sensitive to single scattering albedo of molecule in the ocean comparatively. 


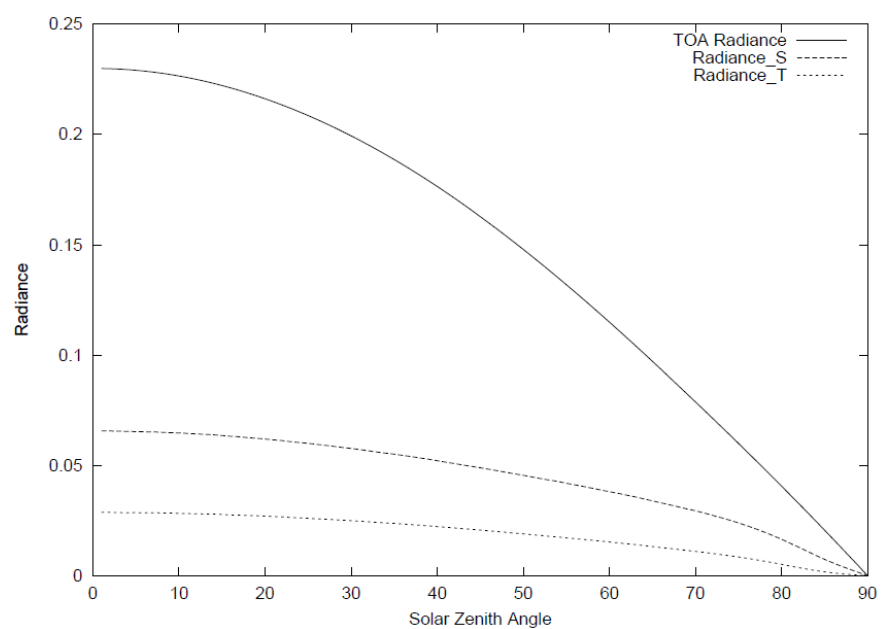

(a)As a function of solar zenith angle

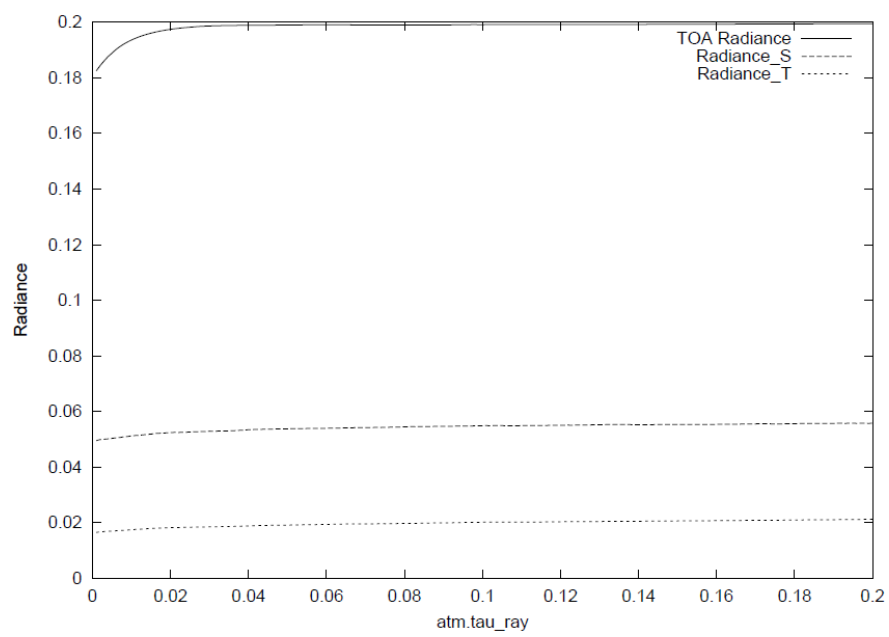

(b)As a function of optical depth of suspended solid

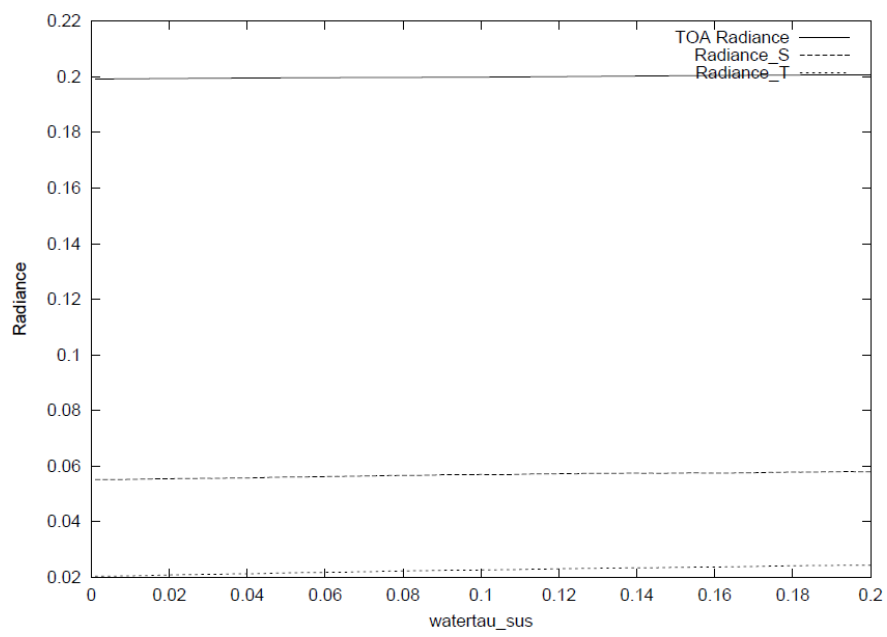

(c)As a function of oceanic molecule (sea water) optical depth

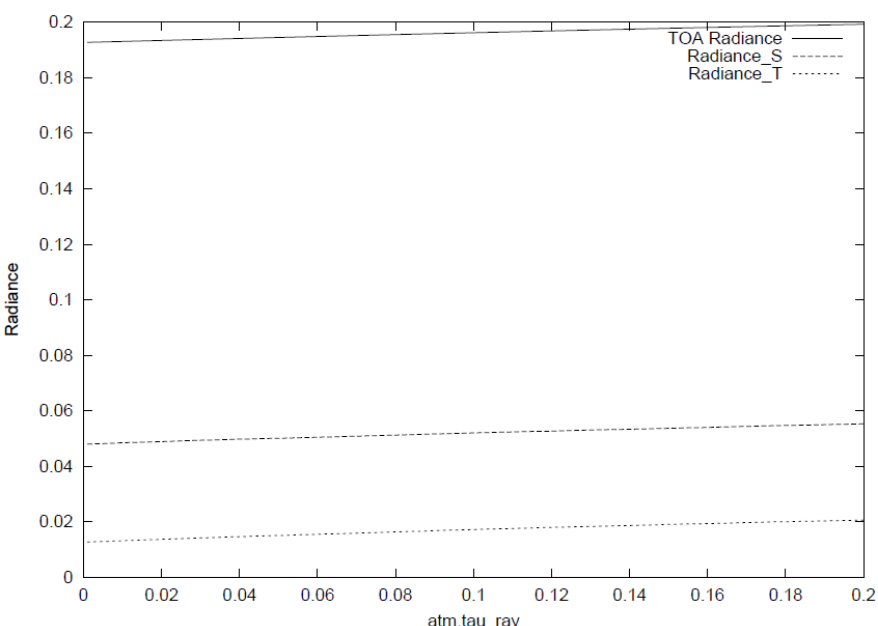

(d)As a function of atmospheric molecule optical depth

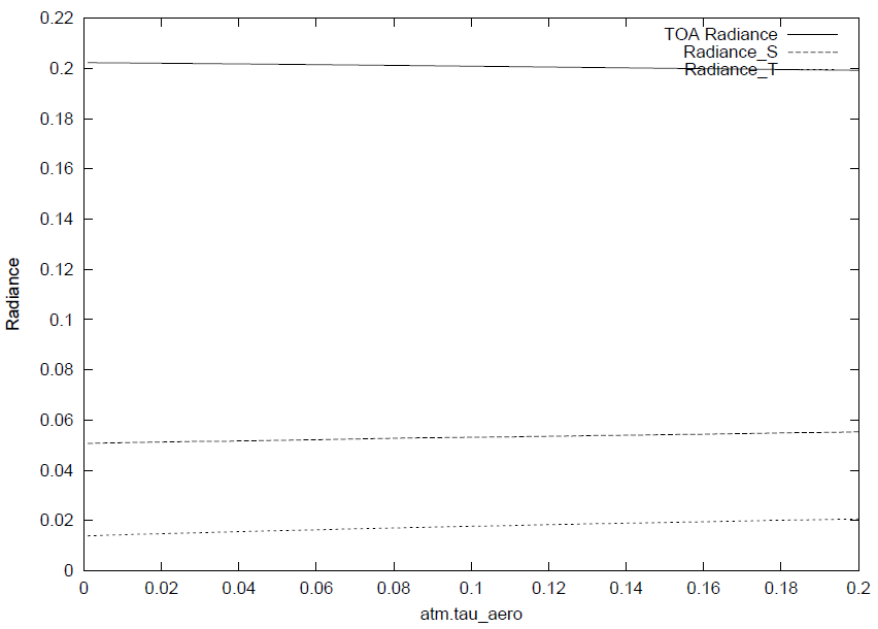

(e)As a function of aerosol optical depth

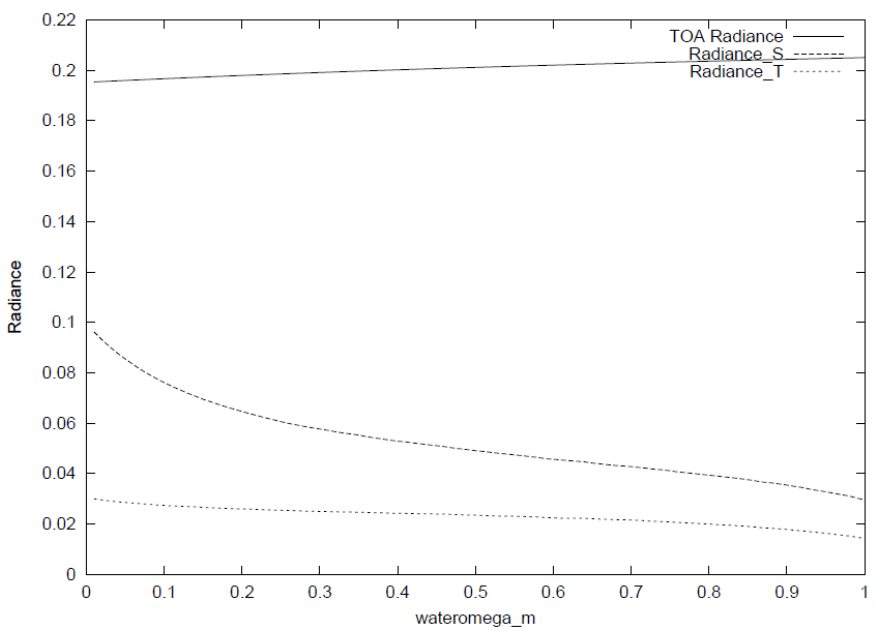

(f)As a function of single scattering albedo of suspended solid 


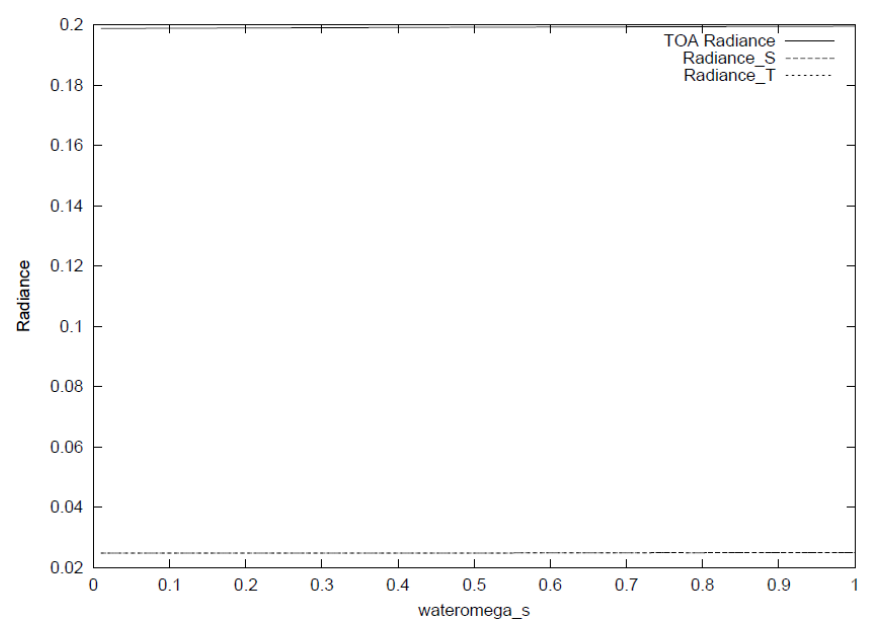

(g)As a function of single scattering albedo of molecule in the ocean (sea water)

Figure 6. TOA radiance, water leaving radiance and TOA radiance excluding the contribution due to scattering in the atmosphere

TOA radiance changes are summarized in Table 1. TOA radiance changes for suspended solid optical depth is greatest followed by sea water optical depth, single scattering albedo of suspended solid, atmospheric molecule optical depth, aerosol optical depth, and single scattering albedo of sea water.

\section{TABLE I. TOA RADIANCE CHANGES}

\begin{tabular}{|l|l|}
\hline Contribution & TOA_radiance_changes \\
\hline Oceanic_molecule_optical_depth & 0.02813 \\
\hline Suspended_solid_optical_depth & 0.1994 \\
\hline Atmospheric_molecule_optical_depth & 0.006545 \\
\hline Aerosol_optical_depth & 0.003067 \\
\hline Oceanic_molecule_single_scattering_albedo & 0.000761 \\
\hline Suspended_solid_single_scattering_albedo & 0.009676 \\
\hline
\end{tabular}

\section{Bi-directional Reflectance Distribution Function: BRDF}

Bi-directional Reflectance Distribution Function: BRDF is estimated with the same default parameters. Solar zenith angle is set at 90 degree. Figure 7 shows the estimated ocean surface reflectance as a function of observation zenith angle. There is sun glint at around 80 degree of observation zenith angle because the solar zenith angle is 90 degree.

\section{CONCLUSION}

Monte Carlo Ray Tracing: MCRT based sensitivity analysis of the geophysical parameters (the atmosphere and the ocean) on Top of the Atmosphere: TOA radiance in visible to near infrared wavelength regions is conducted. As the results, it is confirmed that the influence due to the atmosphere is greater than that of the ocean. Scattering and absorption due to aerosol particles and molecules in the atmosphere is major contribution followed by water vapor and ozone while scattering due to suspended solid is dominant contribution for the ocean parameters.

It is also found that the sensitivity on TOA radiance for suspended solid optical depth is greatest followed by sea water optical depth, single scattering albedo of suspended solid, atmospheric molecule optical depth, aerosol optical depth, and single scattering albedo of sea water.

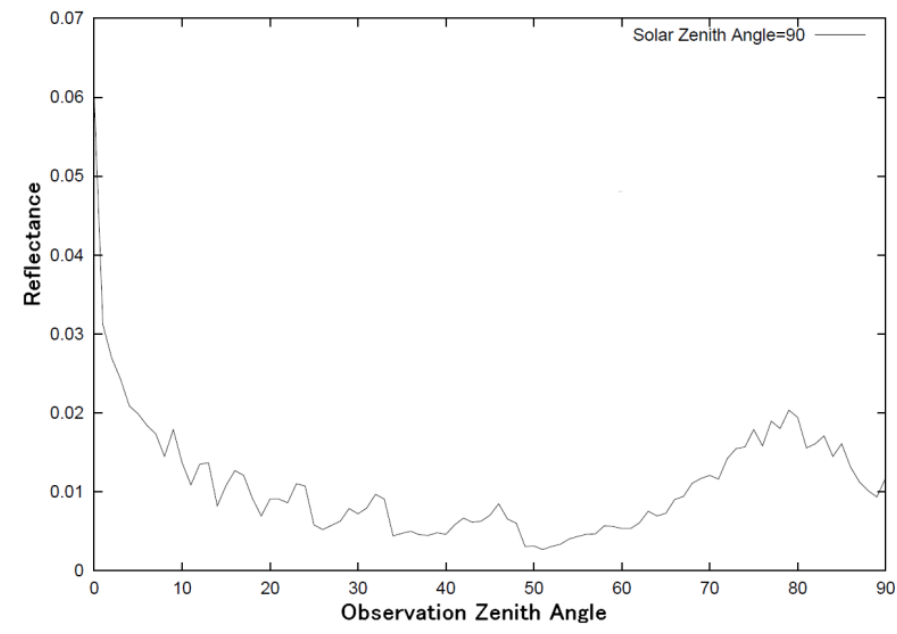

Figure 7. Estimated ocean surface reflectance as a function of observation zenith angle

\section{ACKNOWLEDGMENT}

The author would like to thank Dr. Yasunori Terayama and Mr. Keiji Ono for their contributions to conduct simulation studies.

\section{REFERENCES}

[1] Kohei Arai, Adjacency effect of layered clouds estimated with MonteCarlo simulation, Advances in Space Research, Vol.29, No.19, 18071812, 2002.

[2] Kohei Arai, Lecture Note for Remote Sensing, Morikita Publishing Inc., (Scattering), 2004.

[3] Kohei Arai, Fundamental Theory for Remote Sensing, Gakujutsu-Tosho Publishing Co., Ltd.,(Lambertian), 2001.

\section{AUTHORS PROFILE}

Kohei Arai, He received BS, MS and PhD degrees in 1972, 1974 and 1982, respectively. He was with The Institute for Industrial Science, and Technology of the University of Tokyo from 1974 to 1978 also was with National Space Development Agency of Japan (current JAXA) from 1979 to 1990. During from 1985 to 1987, he was with Canada Centre for Remote Sensing as a Post Doctoral Fellow of National Science and Engineering Research Council of Canada. He was appointed professor at Department of Information Science, Saga University in 1990. He was appointed councilor for the Aeronautics and Space related to the Technology Committee of the Ministry of Science and Technology during from 1998 to 2000. He was also appointed councilor of Saga University from 2002 and 2003 followed by an executive councilor of the Remote Sensing Society of Japan for 2003 to 2005. He is an adjunct professor of University of Arizona, USA since 1998. He also was appointed vice chairman of the Commission "A" of ICSU/COSPAR in 2008. He wrote 30 books and published 332 journal papers. 